\title{
Preference-based algorithms for predicting herbivore diet composition
}

\author{
MF Quirk, JW Stuth \\ Department of rangeland ecology and management, Texas A \& $M$ university, \\ College Station, TX 77843-2126, USA
}

\begin{abstract}
Methods for assessing carrying capacity of grazing lands would be enhanced by reliable predictions of the species composition of diets selected by domestic and wild herbivores. POPMIX, a region-neutral multispecies stocking-rate calculator, predicts diet composition of herbivores based on empirically-derived relationships between the proportions of preferred, desirable, undesirable, non-consumed, and toxic species in the field with their corresponding proportions in the diet. We tested the adequacy of this array of preference categories for describing diet selection of two different herbivores.
\end{abstract}

Eighteen pre-selected grazing paths $(50 \mathrm{~m}$ $\times 2 \mathrm{~m}$ ) were established in Quercus stellata savanna of east-central Texas. In August 1993 and May 1994, extrusa samples were collected from each path by esophageal-fistulated goats and cattle ( 2 head of each species per path ; 20-minute diet collection after fasting), and diet composition determined by a macrofragment technique. Forage species composition of standing crop within each path was measured by combination of the comparative yield and dry-weight-rank procedures of Tothill et al (1992, CSIRO Trop Agron Tech Mem, 78). Ivlev's electivity index, $E$ (which ranges from -1 to 1), was calculated as described by Lechowicz (1982, Oecologia, 52, 22-30) to characterize forage species - within each path, at each sampling time, and for each herbivore type - as belonging to one of the POPMIX preference categories, i.e., preferred $(E>0.1)$,

\section{Forage preference category Animal species}

\begin{tabular}{ll}
\hline Preferred & Cattle \\
Undesirable & Goats \\
& Cattle \\
& Goats
\end{tabular}

desirable $(-0.1<E<0.1)$, undesirable $(E<-0.1)$, and nonconsumed ( $E=-1$ for all occurrences). We then tested relationships between the proportions of the diet represented by forages within a preference category and the corresponding proportions in the field. Relationships for the desirable categories are not discussed as they are linear by definition.

Time of sampling had little affect on diet : field composition relationships within a preference category. The preferred category for cattle showed the strongest predictive value. Lack of fit appeared to be associated with variable strengths of preference for forage species within a preference class. For example, much of the variation in the goat data was associated with strong preference for rarer browse species, or forbs such as Neptunia lutea. When such species were unavailable to goats, diet : field composition relationships conformed to that observed in cattle. These stronger preferences of goats reflect a more selective foraging strategy. Accounting for different foraging strategies, as well as improving the predictability of relationships, may require splitting each of the current preference categories into 2 or 3 subcategories. This would create a family of diet \%: field \% curves within each broad preference category. The challenge then arises whether a knowledgeacquisition protocol can be devised to adequately distinguish subcategories within preference classes to meet the needs for resource management. 